\title{
Visualization of Pre-set Vortices in Boundary Layer Flow over Wavy Surface in Rectangular Channel
}

\author{
A.C. Budiman ${ }^{1 *}$, H. Mitsudharmadi², Y. Bouremel ${ }^{3}$, S.H. Winoto ${ }^{4}$, H.T. Low ${ }^{1}$ \\ ${ }^{1}$ Department of Mechanical Engineering, National University of Singapore, 9 Engineering Drive \\ 1, Singapore 117576 \\ ${ }^{2}$ Reactive Flow Modeling Laboratory Group in Clean Combustion Research Center, King \\ Abdullah University of Science and Technology, Thuwal 23955, Kingdom of Saudi Arabia \\ (formerly at Experimental Aeroscience Group, Temasek Laboratories at National University of \\ Singapore, 5A Engineering Drive 1 \#09-02, Singapore 117411)
}

${ }^{3}$ Experimental Aeroscience Group, Temasek Laboratories at National University of Singapore, 5A Engineering Drive 1 \#09-02, Singapore 117411

${ }^{4}$ Formerly at Department of Mechanical Engineering, National University of Singapore, 9 Engineering Drive 1, Singapore 117576

*Tel: +65-8514 7988

*Email : christantho@nus.edu.sg

\begin{abstract}
Smoke-wire flow visualization is used to study the development of pre-set counterrotating streamwise vortices in boundary layer flow over a wavy surface in a rectangular channel. The formation of the vortices is indicated by the vortical structures on the cross-sectional plane normal to the wavy surface. To obtain uniform spanwise vortex wavelength which will result in uniform vortex size, two types of spanwise disturbances were used: a series of perturbation wires placed prior and normal to the leading edge of the wavy surface, and a jagged pattern in the form of uniform triangles cut at the leading edge. These perturbation wires and jagged pattern induce low velocity streaks that result in the formation of counter-rotating streamwise vortices that evolve downstream to form the mushroom-like structures on the cross-sectional plane of the flow. The evolution of the most amplified disturbances can be attributed to the formation of these mushroom-like structures. It is also shown that the size of the mushroom-like structures depends on the channel entrance geometry, Reynolds number, and the channel gap.
\end{abstract}

Keywords counter-rotating streamwise vortices, wavy surface, boundary layer flow, triangular pattern leading edge, smoke-wire flow visualization, flow instability

\section{Introduction}

Centrifugal instability in concave surface boundary layer flow may result in counter-rotating streamwise vortices, known as Görtler vortices. Such vortices can also be found in a channel made of two wavy (corrugated) surfaces as reported by Nishimura et al. (1990) who found that such vortices mostly occurred for $2 a / H>1$, where $a$ is the amplitude of the corrugation and $H$ the height of the channel. Nishimura et al. (1990) show that the flow from the lower wall arises at a discrete spanwise location and forms a pair of streamwise vortices resembling mushroom-like structures similar to Görtler vortices for the channel flow with amplitude $a$ of $3.5 \mathrm{~mm}$ and wavelength of corrugation $\lambda$ of $28 \mathrm{~mm}$ at Reynolds number $R e$ of 169 , where $R e=U_{a v} H / v$, for which $U_{a v}$ is the average velocity, $H$ the height of the channel, and $v$ the kinematic viscosity of the fluid.

At $R e=300$, the centrifugal instability leads to the formation of counter-rotating streamwise vortices along the whole sinusoidal wavy channel (Gschwind et al., 1995). Gschwind et al. (1995) also established a diagram that proves the existence of a critical or neutral curve in the stability diagram for the transition between laminar to turbulent flow.

The transition from laminar to turbulent flow along a concave surface involves the growth of primary instability, followed by the growth of secondary instability. The primary instability consists of the formation of Görtler-type counter-rotating streamwise vortices (Bahri et al, 1999), while the onset of the secondary instability is indicated by the appearance of the inflectional 
velocity profiles in the boundary layer (Mitsudharmadi et al., 2005). Mitsudharmadi et al. (2005) found that the growth rate of the secondary instability observed was 6.5 times higher than that of the primary instability, which leads to the breakdown of the streamwise structures. Thus, the streamwise vortices do not breakdown directly to turbulence.

In the case of both Couette flow (Floryan, 2001) and Poiseuille flow (Floryan, 2007) over a wavy wall, the wall waviness forces the flow to change direction resulting in the creation of a centrifugal force field whose magnitude is dependent on the amplitude and the wavelength of the wavy surface. Its effect differs from destabilizing over the concave to stabilizing over the convex part of the corrugation (Floryan, 2001). The critical conditions for the instability to occur depend on the combination of the stabilizing and the destabilizing effects of the field (Floryan, 2007). When the amplitude of the corrugation in the channel is smaller than the critical value, the vortices will not be destabilized regardless of the Reynolds number. Similarly, if the amplitude of the corrugation exceeded the well-defined range, the vortices will also not be observed (Asai and Floryan, 2006).

From a linear stability analysis of flow in a channel bounded by wavy walls, Cabal et al. (2002) found that the wall waviness gives rise to instability that generates streamwise vortices. Asai and Floryan (2006) used one wavy wall with dimensionless number $S=a / H=0.02$ (which corresponds to $a=0.03 \mathrm{~mm}$ and wave number $\alpha=1.02$ ) for $\lambda=46.2 \mathrm{~mm}$ and revealed that the critical Reynolds number $R e_{c r}$ decreases by $30 \%$ for the flow in the wavy channel compared to that in a smooth channel.

Since there has been few experimental works on boundary-layer flow over a wavy surface, the current study aims to visualize the effect of wavy surface on the vortices pre-set by means of leading edge pattern. The Floryan's global instability correlation (Floryan, 2007) will be used as a guide to set the minimum corrugation geometry and $R e$ to ensure the instability condition. Additionally, the variation of $R e$ and the channel height to the development of these streamwise vortices will also be investigated.

\section{Description of Experiment}

Two acrylic plates, each plate has a spanwise width of $159 \mathrm{~mm}$, are used in this experiment. One of the plates consists of four wavelengths of sinusoidal surface that connected to flat surface at both ends. This corrugated surface amplitude $a$ and wavelength $\lambda$ is $3.75 \mathrm{~mm}$ and $76 \mathrm{~mm}$, respectively. The entrance flat surface is $150 \mathrm{~mm}$ long while another flat plate on the other end is $230 \mathrm{~mm}$, giving a total channel length of $684 \mathrm{~mm}$. Both plates are covered with smooth black matt film to obtain low-reflection conditions for visualization. These plates were placed with a gap $H=$ $40 \mathrm{~mm}$ into the test section of a small wind tunnel which has a cross-sectional area of $160 \mathrm{~mm} \mathrm{x}$ $160 \mathrm{~mm}$. The free stream turbulent intensity of this wind tunnel is $0.25 \%$ for a velocity range of 1 to $4 \mathrm{~m} / \mathrm{s}$.

There are three different configurations used in this experiment, based on how the corrugated surface is connected to the entrance flat plate (Fig. 1). The first (Fig. 1(a)) is called Peak First (PF), for which the flat entrance channel is connected to the peak of corrugated surface. The second (Fig. 1(b)) is called From Origin (FO), for which the flat plate is placed in the center of axis, and the third (Fig. 1(c)), which is called Valley First (VF) design, is set by placing the valley of corrugated surface next to the flat plate. Different from earlier configurations, in the VF design the flow will initially be destabilized by the concave part of the corrugated channel.

Flow visualization was carried out by means of smoke-wire technique. In this technique, a nickel-chromium alloy resistance heating wire of $0.193 \mathrm{~mm}$ diameter was placed parallel to the flat surface part upstream of the corrugated surface at about $1 \mathrm{~mm}$ from the surface and $90 \mathrm{~mm}$ upstream of the first peak of the corrugated surface. Paraffin oil from a pressurized container was allowed to drip along the smoke-wire to coat it completely. A direct current (DC) power supply that provides current in the range of 1 to 1.3 Amperes and voltage ranging from 11 to 18 Volts was used to heat the paraffin oil coated wire that results in a thin layer of smoke. The required DC power depends on the air flow velocity; the higher the velocity the higher the power required to generate streaks of smoke. The wire Reynolds number $R e_{d}$ is about 50 for airflow velocity of 4 $\mathrm{m} / \mathrm{s}$ to avoid the formation of von Karman vortex-street downstream of the smoke-wire. A green laser light (of wavelength $=532 \mathrm{~nm}$ ) and power of $50 \mathrm{~mW}$ was used to create a laser sheet on the cross-section view of the flow. The visualizations were carried out by creating a laser sheet at a certain distance after the leading edge, the first peak, second peak, and the second valley (between the first and second peak). All these spanwise images were recorded by a camera placed downstream of the wind tunnel test section. Top image was taken using LED illumination instead 
of using green laser sheet. The schematic diagram of the visualization set-up and the location of these peaks and valleys are shown in Fig. 2.

\section{Results and Discussion}

In the experiments, naturally generated counter-rotating streamwise vortices usually have nonuniform spanwise wavelengths unless great care is taken for the surface finish and careful control is imposed on the flow condition, as in the experiment done by Wortmann (1969). The nonuniformity in vortex wavelength and hence vortex size causes difficulty and bias in studying their development. Consequently, a method to pre-set the vortex wavelength is required.

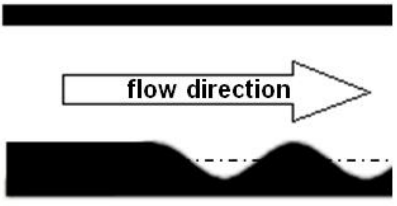

(a) Peak First (PF)

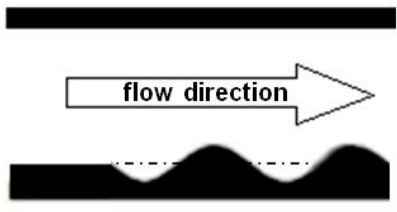

(b) From Origin (FO)

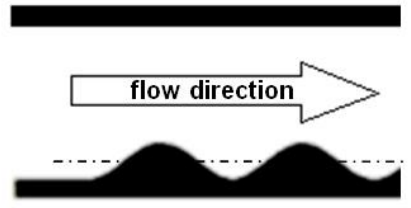

(c) Valley First (VF)

Fig. 1 Sketch of corrugated channel entrance geometries: (a) Peak First (PF), (b) From Origin (FO), and (c) Valley First (VF)

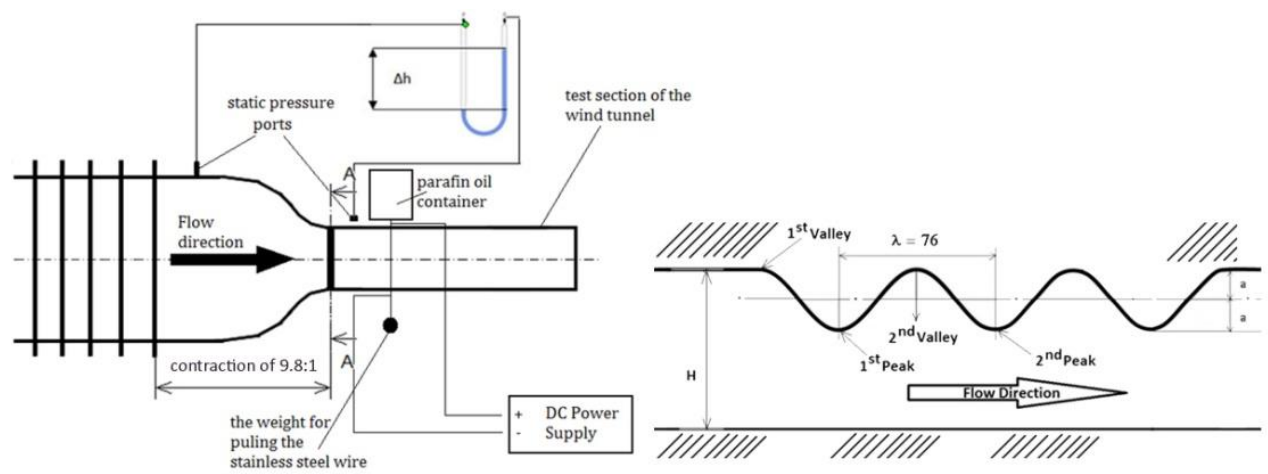

Fig. 2 Sketch (not to scale) of the wind tunnel with the instrument devices used for flow visualization and of VF-type corrugated wall in the test section (units are in $\mathrm{mm}$ )

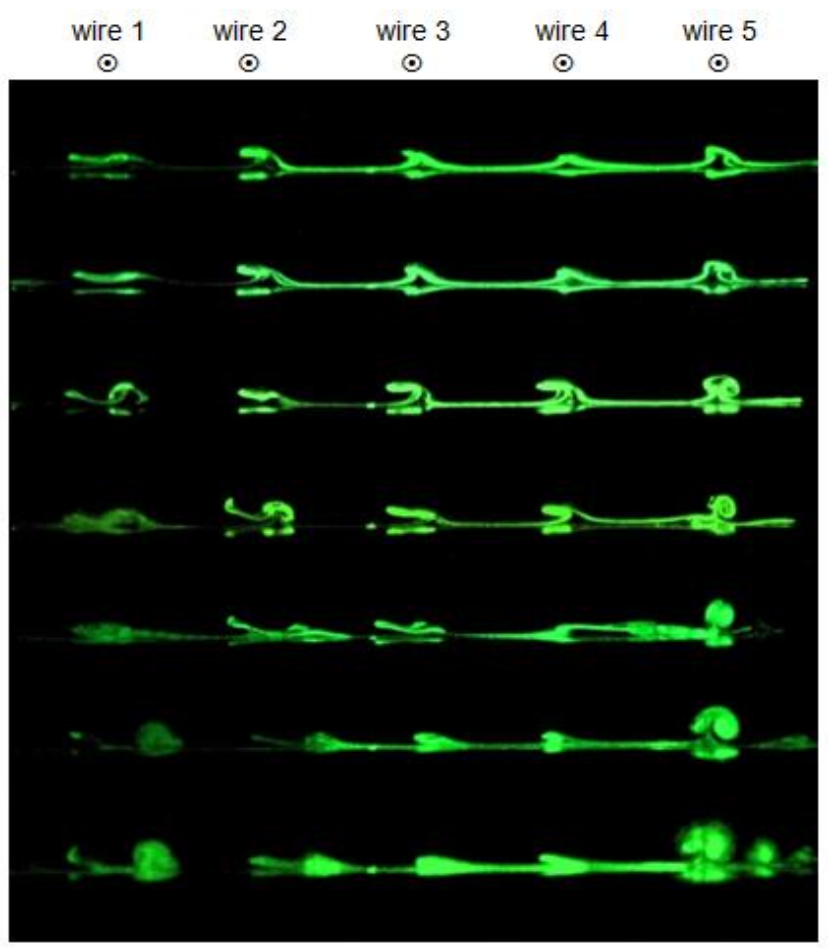

Fig. 3 Evolution of the vortices induced by a series of 5 perturbation wires of diameter $0.7 \mathrm{~mm}$ for $R e=2500$, $3500,4250,5500,6250,6500$, and 7000 (top to bottom) at the first peak of corrugation with $S=0.250$ 
As a preliminary work to pre-set the spanwise wavelength of the vortices, the method used by Ajakh et al. (1999), Peerhossaini and Bahri (1998), Toe et al. (2002), and Mitsudharmadi et al. (2004) was adopted. In this case, five perturbation wires of $0.7 \mathrm{~mm}$ diameter were installed normal to the entrance flat plate with the spanwise spacing of $14 \mathrm{~mm}$ from each other. A commercially available corrugated plate with $a=7.5 \mathrm{~mm}$ was used for this preliminary study. The flow velocity was gradually increased from $1.3 \mathrm{~m} / \mathrm{s}$ to $3.6 \mathrm{~m} / \mathrm{s}$ at constant $S=0.250$. These conditions correspond to $R e$ from 2500 to 7000 . The corrugated surface entrance geometry used in this study was a VF type. The perturbation wires would induce the formation of streaks of low velocity region behind each wire and high velocity region in the spacing between wires. It results in spanwise modulation of the streamwise velocity $U$ with the wavelength similar to the spanwise spacing between the wires. The boundary layer thicknesses at the high velocity regions are thinner than those at the low velocity regions. This agrees well with quantitative velocity-field measurements using hot wire anemometry in boundary layer flow over the concave surface reported by Mitsudharmadi et al. (2004).

The preliminary results presented in Fig. 3 show that as Re increased to 3500, the occurrence of the wavy pattern are evident downstream of each perturbation wires. The formation of five peaks along the spanwise can be attributed to the evolution of the disturbance within the linear region (Mitsudharmadi et al., 2004). This phenomenon suggests the formation of one pair of counter-rotating vortices downstream of each perturbation wires. The wavy pattern transformed into mushroom-like structures that become prominent in the range of $3500<R e<7000$. Within this range, the formation of the stem and the head of the mushroom are visible, especially at the spanwise location aligned with the first and the fifth perturbation wires. Downstream of the other three wires, the mushroom-like structures are not clearly seen. This could be due to the nonuniform smoothness of the corrugated surface in the spanwise direction that may result in different amplification rate of disturbances. The vertical line at the center of the vortex is defined as the "stem" of this mushroom-like structure. This stem corresponds to the low momentum fluid that moves away from the wall surface in the region called "upwash" where the boundary layer is thicker and shear stress is lower. Since this low momentum fluid cannot "penetrate" the high momentum fluid layer in the middle of the channel, it returns back toward the wall at "downwash" region and develops the "mushroom hat" pattern. The occurrence of downwash and upwash regions generates wavy pattern at the edge of the boundary layer (Mitsudharmadi et al., 2004). At $R e=7000$ the appearance of the mushroom-like structures is diffused prior to turbulence.

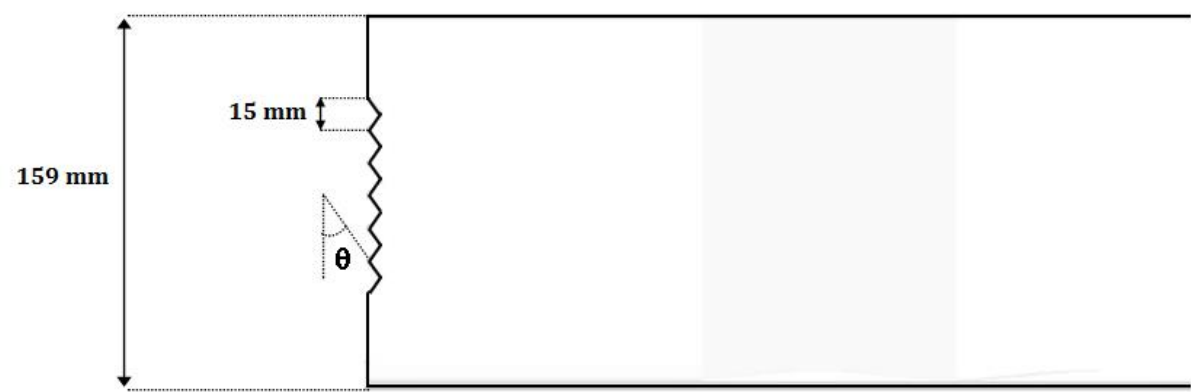

Fig. 4 Schematic design of jagged cut pattern at the leading edge to create uniform spanwise disturbances

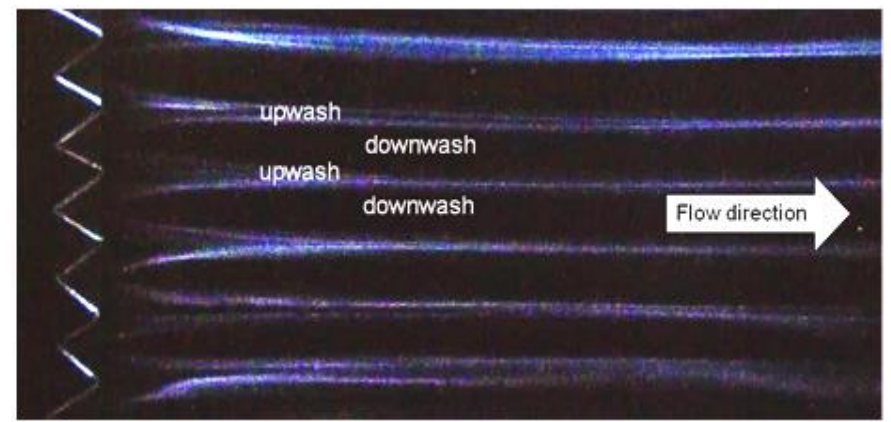

Fig. 5 Linear smoke streaklines from the top view of the entrance flat plate before the corrugation at $R e=$ 3500. Air is flowing from left to right 


\subsection{Jagged leading edge to substitute the perturbation wires}

Although prominent mushroom-like structure is discernible by installing five perturbation wires, the expected appearance of five pair of vortices with uniform spanwise wavelength cannot be found. Therefore, the perturbation wires were replaced by jagged cut pattern at the leading edge of the entrance flat plate before the corrugated surface (Fig. 4). The acrylic plates with $a=3.75$ $\mathrm{mm}$ were fabricated and the gap $H$ was increased to $40 \mathrm{~mm}$, giving $S=0.094$. This jagged pattern consists of six isosceles triangles with base length of $15 \mathrm{~mm}$ and base angle $\theta$. The triangle edges and its tip forces the fluid to be driven upward from the surface, creating mushroom's stem (upwash region) and returns back to form a mushroom hat with maximum shear (Fig. 5). This method has been successfully used to generate prominent vortices with comparable spanwise wavelength (Budiman et al., 2013). The straight streaklines depicted in Fig. 5 is associated to the linear disturbance growth rate region, as reported by Mitsudharmadi et al. (2004).

Using jagged leading edge with $\theta=40^{\circ}$, six mushroom-like structures are visible on the first peak of corrugation at $R e=4750,5750$, and 6800 for $S=0.094$ (Fig. 6). The corrugated surface entrance geometry used in this section is VF type, which is similar to that in the preliminary work. Qualitatively, it can be said that all structures were relatively uniform in spanwise wavelength and also shape. Unlike the vortices induced by the perturbation wires, these vortices have long, and distinct mushroom stems.

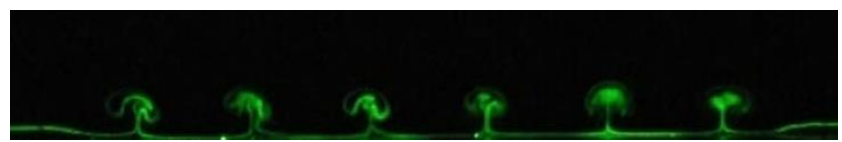

(a)

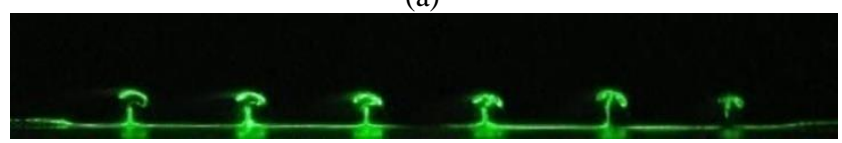

(b)

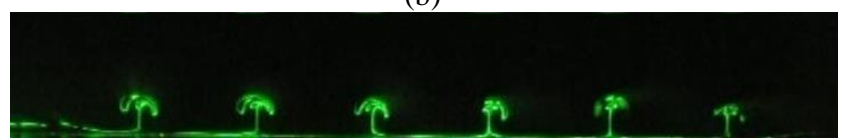

(c)

Fig. 6 Evolution of the vortices induced by a jagged leading edge at the first peak of corrugation with $S=$ 0.094, VF-type configuration: (a) at $R e=4750$, (b) at $R e=5750$, and (c) at $R e=6800$

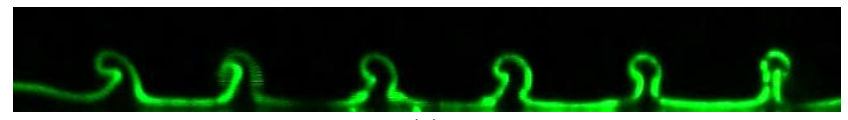

(a)

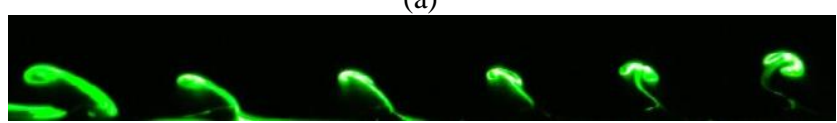

(b)

Fig. 7 Counter-rotating streamwise vortices found at the first peak for $R e=4500$ and $S=0.125$ show the effect of base angle of the triangle leading edge: (a) for $\theta=40^{\circ}$ and (b) for $\theta=60^{\circ}$

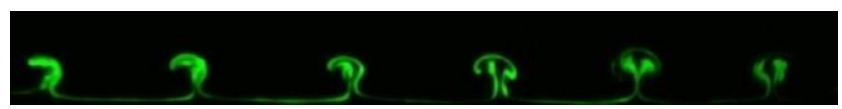

(a)

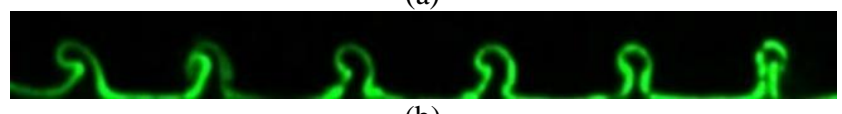

(b)

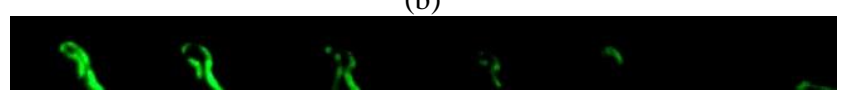

(c)

Fig. 8 Counter-rotating streamwise vortices found at the first peak for $R e=4500$ and $S=0.125$ show the effect of channel entrance configuration: (a) for Valley First, (b) From Origin, and (c) Peak First type 
Figure 7 shows the different shape of vortex structures as the base angle $\theta$ was changed to $60^{\circ}$. Qualitatively, the appearance of the mushroom hats and stems are more prominent than those of $\theta$ $=40^{\circ}$. It is also shown that the stems are no longer straight, indicating that these equilateral triangles at the leading edge produce stronger disturbance with possible higher growth rate.

\subsection{Effect of different channel entrance geometries and $S$}

According to Floryan's global critical stability conditions, the instability occurs if the corrugated surface contains an "active" corrugation wave number (Floryan, 2007). It is demonstrated through the visualization of the vortices at the first peak for different corrugated surface entrance geometries that the evolution of the vortices is different due to the $\mathrm{VF}$, FO, and PF entrance geometry types. As shown in Fig. 8, at the first peak of corrugation, the vortices due to the FO type are relatively smaller, while for PF entrance configuration, only two vortices with very small mushroom hat on the left are visualized clearly. This might be due to the stabilizing and destabilizing effect from the convex and concave surface, respectively (Floryan, 2001). For VF type, the induced disturbances will not be stabilized until they reach the first peak location. In contrast, due to the PF entrance type, the induced disturbances will encounter the stabilizing effect first, thus it may reduce its growth rate so the structure formed becomes smaller.

Spanwise variation of the mushroom-like structures at the first peak or corrugation induced by jagged leading edge with $\theta=60^{\circ}$ for FO entrance type and two different values of $S$ at $R e=5500$ is presented in Fig. 9. Two different gaps of the channel $\mathrm{H}$ were used to change the value of $S$. A relatively bigger size of mushroom hat and the breakdown of the rightmost vortex at $S=0.125$ ( $H$ $=30 \mathrm{~mm}$ ) in Fig. 9(b) indicate that the flow becomes more unstable as $S$ increases. To summarize, the spanwise structure variation formed by vortex pair due to various disturbances, $R e$, and channel geometry plotted in the Floryan's instability chart is presented in Fig. 10. Since all experiments were performed in the unstable region, it is hypothesized that the flow is destabilized due to the evolution of the induced disturbances where its stability condition is affected by the variation of $R e$ and $S$.

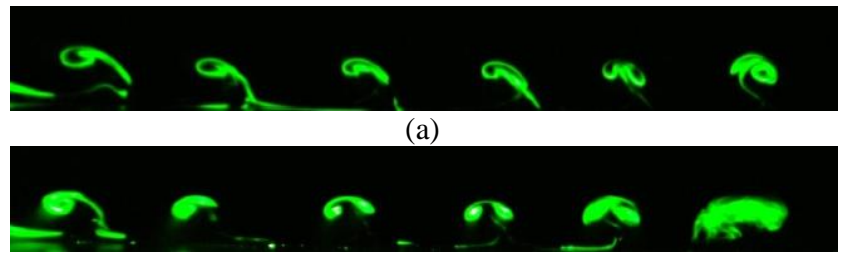

(b)

Fig. 9 Counter-rotating streamwise vortices found at the first peak for $R e=5500$ show the effect of channel gap for the same corrugation amplitude, (a) for $S=0.094$ and (b) for $S=0.125$

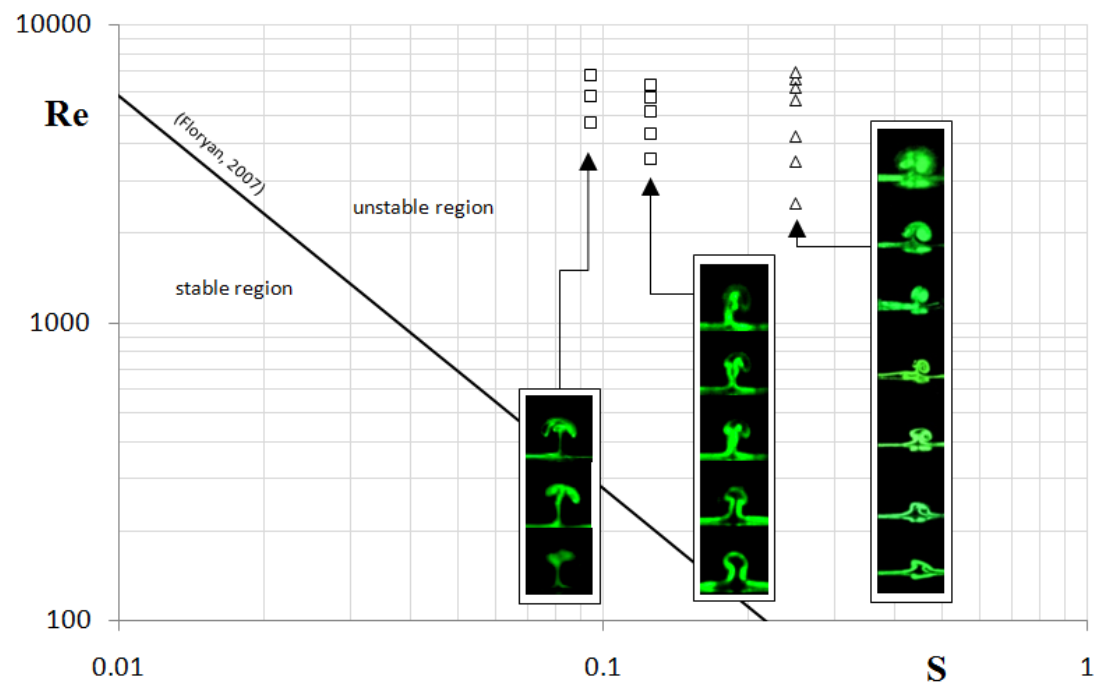

Fig. 10 Images of single vortex for various Re and $S$, plotted with the global instability line reported by Floryan (2007) as the reference for stable and unstable region. $\Delta$ refers to the image obtained from experiments using perturbation wires. $\square$ indicates present works using triangle leading edge to pre-set the spanwise wavelength of vortices, VF-type $40^{\circ}$ triangle (left) and FO-type $40^{\circ}$ triangle (right) 


\subsection{The development of counter-rotating streamwise vortices}

As the fluid passed the jagged leading edge, disturbances are induced, from which the most amplified one will develop downstream. Figure 11 shows the vortices created by the jagged leading edge for $\operatorname{Re}=4300$ and $S=0.125$ at four different streamwise positions: at the flat surface $5 \mathrm{~cm}$ downstream from the leading edge, the first peak, second valley, and second peak. There are in total six structures are observed and it seems that for $R e=4300$ and $S=0.125$, the base distance of each triangles is significant to prevent the interaction between two adjacent vortices. However, this kind of interaction might affect the development of the vortices if the flow conditions are more unstable, as shown earlier in Fig. 7(b).

When the flow passes over the concave part of the wavy surface right before the first peak, the induced disturbances will be more amplified by the centrifugal instability. It is indicated by the appearance of longer mushroom stem as it reached the first peak of corrugation. After passing through that concave part, the vortices were lifted up above the separation bubble which is formed in the valley of corrugation. As a result, the stem of the vortices that supports the mushroom hat turns out to be weaker as it moves downstream so that the vortex structures are vulnerable to the free-stream flow (Tandiono et al., 2009). Finally at the second peak, more disturbed structures were found. Further investigation showed that after the second peak, the mushroom-like structures were not clearly defined, indicating that the structures have broken down prior to turbulence.

\section{Conclusion}

Counter-rotating streamwise vortices as indicated by vortical structures on the cross-sectional planes normal to the corrugated wall have been visualized by smoke-wire flow visualization technique. The spanwise spacing of these vortices has been successfully controlled within the unstable region of the Floryan's global instability chart (Floryan, 2007) by introducing spanwise perturbation generated by a series of thin wires installed prior to and normal to the wavy wall and also by means of uniform triangles cut on the leading edge of the corrugated wall. It reveals that the most amplified disturbances would result in prominent counter-rotating streamwise vortices in the form of mushroom-like structures.

Various channel entrance geometry and amplitude-to-channel gap ratio $S=a / H$ have been tested experimentally. It is found that the Valley First (VF) configuration, which allows the flow to enter the concave surface first, generates relatively larger vortices. The size of the structures here represents stronger amplification of the disturbances. In terms of $S$, this flow visualization agrees with the analytical solution (Floryan, 2007), which said that as $S$ increases, the flow becomes more unstable.

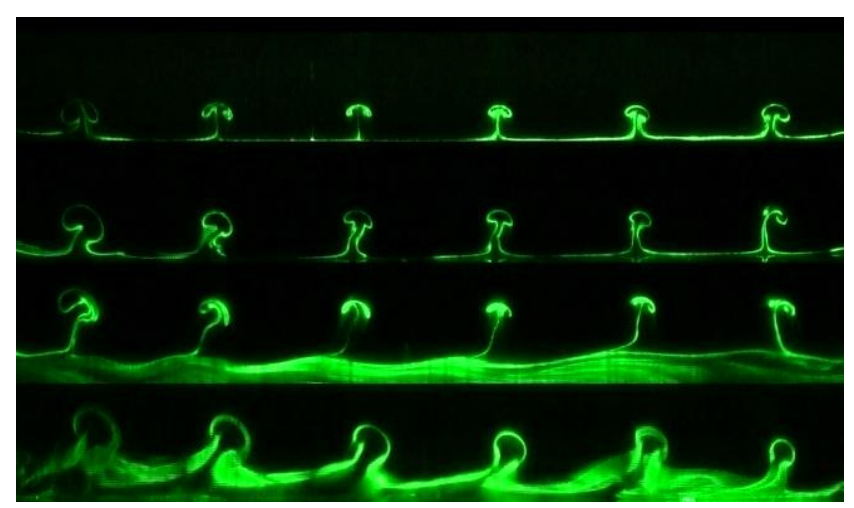

Fig. 11 Evolution of the vortices induced by jagged leading edge. Images were taken from various locations, from top to bottom: at $5 \mathrm{~cm}$ after the jagged leading edge, the first peak, second valley, and second peak of corrugation for $R e=4300$ and $S=0.125$

\section{Acknowledgment}

The authors would like to express their sincere gratitude to the Temasek Laboratories, National University of Singapore for the opportunity to conduct the experiments at its Aeroscience 
Laboratory, and also to thank Mr. Sim Lucius and Mr. M. N. Adha b Jamaludin for their help in setting up the experimental rig and in collecting the experimental data. The first author is a recipient of NUS Research Scholarship and would also like to thank the AUN/SEED-Net.

\section{References}

Ajakh A, Kestoras MD, Toe R, and Peerhossaini H (1999) Influence of forced perturbation in the stagnation region on Görtler instability. AIAA J 37(12):1572-1577.

Asai M and Floryan JM (2006) Experiments on the linear instability of flow in a wavy channel. Eur J Mech B-Fluid 25:971-986.

Bahri F, Kohama Y, Iino J, and Aota (1999) Effect of the pressure gradient on the secondary instability of Görtler flow. In: Fasel HF and Saric WS. Laminar-Turbulent Transition-IUTAM Symposium Sedona/AZ, Springer, pp. 535-539.

Budiman AC, Mitsudharmadi H, Low HT, and Winoto SH (2013) Visualization of counter-rotating streamwise vortices in a rectangular channel with one-sided wavy surface. Proceeding of the $31^{\text {st }}$ AIAA Applied Aerodynamics, paper number AIAA 2013-2533.

Cabal A, Szumbarski J, and Floryan JM (2002) Stability of flow in a wavy channel. J Fluid Mech 457:191-212.

Floryan JM. (2001) Centrifugal instability of Couette flow over a wavy wall. Phys Fluids 14(1): 312-322.

Floryan, JM (2007) Three-dimensional instabilities of laminar flow in a rough channel and the concept of hydraulically smooth wall. Eur J Mech B-Fluid 26:305-329.

Gschwind P, Regele A, and Kottke V (1995) Sinusoidal wavy channels with Taylor-Görtler vortices. Exp Therm Fluid Sci 11:270-275.

Mitsudharmadi H, Winoto SH, and Shah DA (2004) Development of boundary-layer flow in the presence of forced wavelength Görtler vortices. Phys Fluids 16(11):3983-3996.

Mitsudharmadi H, Winoto SH, and Shah DA (2005) Secondary instability in forced wavelength Görtler vortices. Phys Fluids 17: 074104.

Nishimura T, Yano K, Yoshino T, and Kawamura Y (1990) Occurrence and structure of TaylorGörtler vortices induced in two-dimensional wavy channels for steady flow", J Chem Eng Jpn 23(6):697-703.

Peerhossaini H and Bahri F (1998) On the spectral distribution of the modes in non-linear Görtler instability. Exp Therm Fluid Sci 16(3):195-208.

Tandiono, Winoto SH, and Shah DA (2009) Visualizing shear stress in Görtler vortex flow. J Visualization 12:195-202.

Toe R, Ajakh A, and Peerhossaini H (2002) Heat transfer enhancement by Görtler instability. Int J Heat Fluid Fl 23:194-204.

Wortmann, F. X. (1969) Visualization of transition. J Fluid Mech 38(3):473-480. 